\title{
Remark on the Abstract Dirichlet Problem for Baire-One Functions
}

\author{
by \\ Ondřej F. K. KALENDA \\ Presented by Czesław BESSAGA
}

Summary. We study the possibility of extending any bounded Baire-one function on the set of extreme points of a compact convex set to an affine Baire-one function and related questions. We give complete solutions to these questions within a class of Choquet simplices introduced by P. J. Stacey (1979). In particular we get an example of a Choquet simplex such that its set of extreme points is not Borel but any bounded Baire-one function on the set of extreme points can be extended to an affine Baire-one function. We also study the analogous questions for functions of higher Baire classes.

1. Introduction. The abstract Dirichlet problem is a question of the following type. Let $X$ be a compact convex subset of a locally convex space and $f$ be a function defined on ext $X$, the set of all extreme points of $X$. Can $f$ be extended to an affine function on $X$ which shares given properties of $f$ ?

A classical theorem of Bauer (see e.g. [2, Theorem 3]) says that any bounded continuous function on $\operatorname{ext} X$ can be extended to a continuous affine function on $X$ if and only if $X$ is a Choquet simplex and $\operatorname{ext} X$ is closed in $X$.

We investigate the analogous questions for bounded Baire-one functions (a function is Baire-one if it is a pointwise limit of a sequence of continuous functions). If $X$ is a Choquet simplex and ext $X$ is $F_{\sigma}$ in $X$ then any bounded Baire-one function on ext $X$ can be extended to an affine Baire-one function on $X$ (see e.g. [15, Theorem 37]). It was conjectured in [8] that the converse holds as well. Recently Spurný [17, Theorem 2] proved that the converse is

2000 Mathematics Subject Classification: 46A55, 54C30, 54H05.

Key words and phrases: Choquet simplex, affine Baire-one function, abstract Dirichlet problem, extreme points, Lindelöf space, $H$-set.

The work is a part of the research project MSM 0021620839 financed by MSMT and partly supported by the research grant GAČR 201/03/933. 
true within metrizable simplices (even in a more general context of simplicial function spaces). However, outside metrizable spaces the converse is not true due to [17, Example 3].

In the present paper we study this problem within a class of simplices introduced by Stacey in [19]. As an application we show in particular that there is a Choquet simplex $X$ with ext $X$ non-Borel such that any bounded Baire-one function on ext $X$ can be extended to an affine Baire-one function on $X$. We also suggest the following conjecture which should replace the previous one of $[8]$.

Conjecture 1. Let $X$ be a convex compact subset of a locally convex space. The following are equivalent.

(1) $X$ is a Choquet simplex and ext $X$ is a Lindelöf $H$-set.

(2) Any bounded Baire-one function on ext $X$ can be extended to an affine Baire-one function on $X$.

$H$-sets are defined in $[10, \S 12, \mathrm{II}]$, where their basic properties are described. Let us recall some equivalent definitions. A set $A \subset X$ is an $H$-set if for any nonempty $B \subset X$ there is a nonempty relatively open $U \subset B$ such that either $U \subset A$ or $U \cap A=\emptyset$. It is clear that the $H$-sets form an algebra containing all open sets. Further, $A$ is an $H$-set in $X$ if and only if $A$ is the union of a scattered family of sets of the form $F \cap G$ with $F$ closed and $G$ open. Recall that a family $\mathcal{U}$ is scattered if it is disjoint and for each nonempty $\mathcal{V} \subset \mathcal{U}$ there is some $V \in \mathcal{V}$ relatively open in $\cup \mathcal{V}$. Or, equivalently, there are an enumeration $\left\{U_{\alpha}: \alpha<\lambda\right\}$ of $\mathcal{U}$ (for a suitable ordinal $\lambda$ ) and open sets $G_{\alpha}, \alpha<\lambda$, such that $U_{\alpha} \subset G_{\alpha} \backslash \bigcup_{\beta<\alpha} G_{\beta}$ for each $\alpha<\lambda$.

If $X$ is a compact metrizable space (or, more generally, a completely metrizable space), then $A \subset X$ is an $H$-set if and only if it is simultaneously $F_{\sigma}$ and $G_{\delta}$. (The "if" part follows from the Baire category theorem, the "only if" part follows from the Montgomery lemma [13, Lemma 16.2].)

It follows from [17] and the previous paragraph that our conjecture is true within metrizable compact convex sets (recall that ext $X$ is $G_{\delta}$ in $X$ whenever $X$ is metrizable). Further, our Theorem 3 below proves the conjecture within Stacey's class of simplices.

After this paper had been finished, Spurný [18] showed that Conjecture 1 is valid within simplices with Lindelöf set of extreme points.

Another conjecture due to Spurný concerns a weaker problem.

Conjecture 2. Let $X$ be a convex compact subset of a locally convex space. The following are equivalent.

(1) $X$ is a Choquet simplex and ext $X$ is Lindelöf.

(2) Any bounded continuous function on ext $X$ can be extended to an affine Baire-one function on $X$. 
The implication $(1) \Rightarrow(2)$ holds true by [8]. In our Theorem 2 we show that the converse holds within Stacey's class of simplices.

In the final section we study the analogous questions for functions of higher Baire classes and show that the situation within Stacey's class of simplices is quite simple.

Before stating our results let us recall some terminology concerning compact convex sets. All topological spaces considered in this paper are supposed to be Hausdorff.

Let $X$ be a compact space. By $P(X)$ we denote the set of all Radon probability measures on $X$ endowed with the weak* topology. (Recall that the dual space $\mathcal{C}(X)^{*}$ is by Riesz's theorem identified with the set of all finite signed Radon measures on $X$ and hence $P(X) \subset \mathcal{C}(X)^{*}$.) If $\mu \in P(X)$ and $f: X \rightarrow \mathbb{R}$ is a $\mu$-measurable function we set $\mu(f)=\int_{X} f d \mu$.

Now suppose that $X$ is a compact convex subset of a locally convex space. A point $x \in X$ is a barycenter of $\mu \in P(X)$ if $f(x)=\mu(f)$ for each affine continuous function $f: X \rightarrow \mathbb{R}$. Any $\mu \in P(X)$ has a unique barycenter which we denote by $r(\mu)$. A function $f: X \rightarrow \mathbb{R}$ is said to satisfy the barycentric formula if it is universally measurable and $\mu(f)=f(r(\mu))$ for each $\mu \in P(X)$.

If $x \in X$, we say that a measure $\mu \in P(X)$ is a representing measure for $x$ if $x=r(\mu)$. The classical Choquet-Bishop-de Leeuw theorem (see e.g. [1, Theorem I.4.8]) says that for any $x \in X$ there is a measure representing $x$ which is maximal in the Choquet ordering. (Recall that $\mu \prec \nu$ in the Choquet ordering if $\mu(f) \leq \nu(f)$ for each convex continuous function $f: X \rightarrow \mathbb{R}$.) If this maximal representing measure is unique for each $x \in X$, the set $X$ is called a Choquet simplex (or, briefly, a simplex). In this case we denote by $\delta_{x}$ the unique maximal measure representing $x$. The Dirac measure supported at $x$ is denoted $\varepsilon_{x}$.

If $f$ satisfies the barycentric formula, it is clearly affine. Conversely, affine continuous functions satisfy the barycentric formula by the definition of barycenter. Further, any affine Baire-one function on $X$ is bounded and satisfies the barycentric formula (see e.g. [1, Theorem I.2.6]). This is not the case for general affine functions (even for Baire-two functions).

2. Stacey simplices. We start by defining the class of simplices we will investigate. This class was introduced in [3, Section VII] to show that the set of extreme points of a convex compact space can have very bad descriptive properties. Later Stacey [19, Theorem 3] showed that these compact convex sets are in fact Choquet simplices.

Let $K$ be any compact space and $A$ any subset of $K$. Further, for any $a \in A$ let $T_{a}$ be a locally compact space consisting of at least two points. For formal reasons suppose that $T_{a} \cap K=\emptyset$ and set $T_{k}=\emptyset$ for $k \in K \backslash A$. 
Let

$$
K^{\#}=\bigcup_{k \in K}\{k\} \times\left(\{k\} \cup T_{k}\right) .
$$

Similarly, for any $M \subset K$ define

$$
M^{\#}=\bigcup_{k \in M}\{k\} \times\left(\{k\} \cup T_{k}\right) .
$$

We equip $K^{\#}$ with the following topology. If $a \in A$ and $t \in T_{a}$, then a neighborhood bases of $(a, t)$ is formed by the sets $\{a\} \times U$ where $U$ is a neighborhood of $t$ in $T_{a}$. A neighborhood base of $(k, k)$ for $k \in K$ is formed by the sets

$$
(U \backslash\{k\})^{\#} \cup\{k\} \times\left(\{k\} \cup T_{k} \backslash H\right)
$$

where $U$ is a neighborhood of $k$ in $K$ and $H \subset T_{k}$ is compact. With this topology $K^{\#}$ is compact.

The set $\{(k, k): k \in K\}$ is a subset of $K^{\#}$ homeomorphic to $K$. We will identify these sets. We will also identify any $M \subset K$ with $\{(k, k): k \in M\}$. Further notice that $\{k\} \times T_{k}$ is canonically homeomorphic to $T_{k}$ and $\{k\} \times\left(\{k\} \cup T_{k}\right)$ is compact for each $k \in K$.

For each $a \in A$ fix a non-Dirac Radon probability measure $\mu_{a}$ on $\{a\} \times T_{a}$ with compact support. Set

$$
\mathcal{A}=\left\{f \in \mathcal{C}\left(K^{\#}\right):(\forall a \in A)\left(f((a, a))=\mu_{a}(f)\right)\right\}
$$

and

$$
X=\left\{\xi \in \mathcal{A}^{*}:\|\xi\|=1 \& \xi(1)=1\right\} .
$$

Then $X$ is a convex weak* compact set. Moreover, it is a Choquet simplex by [19, Theorem 3]. In fact, in [19] this simplex was defined in a different way. Let us show that our definition is equivalent.

For $x \in K^{\#} \backslash A$ set $\mu_{x}=\varepsilon_{x}$. It is shown in [19] that for any $f \in \mathcal{C}\left(K^{\#}\right)$ the set $\left\{x \in K^{\#}: f(x) \neq \mu_{x}(f)\right\}$ is countable and hence $x \mapsto \mu_{x}(f)$ is a Borel function. Moreover, it is shown that the subspace

$$
M=\left\{\nu \in \mathcal{C}\left(K^{\#}\right)^{*}:\left(\forall f \in \mathcal{C}\left(K^{\#}\right)\right)\left(\int_{K^{\#}} \mu_{x}(f) d \nu(x)=0\right)\right\}
$$

is weak ${ }^{*}$ closed in $\mathcal{C}\left(K^{\#}\right)^{*}$, and if $\pi_{M}$ denotes the quotient mapping of $\mathcal{C}\left(K^{\#}\right)^{*}$ onto $\mathcal{C}\left(K^{\#}\right)^{*} / M$, then $\pi_{M}(P(K))$ is a Choquet simplex. To see that this simplex is our $X$ it is enough to show that $M=\mathcal{A}^{\perp}$. The inclusion " $\subset$ " is obvious. The other one follows by the bipolar theorem as $M$ is weak* closed and $M_{\perp}=\mathcal{A}$.

Indeed, the inclusion " $\supset$ " is obvious. The opposite one follows from the fact that $\varepsilon_{a}-\mu_{a} \in M$ for each $a \in A$. To see it let $f \in \mathcal{C}\left(K^{\#}\right)$ be arbitrary. We have 


$$
\begin{aligned}
\int_{K^{\#}} \mu_{x}(f) d\left(\varepsilon_{a}-\mu_{a}\right)(x) & =\int_{K^{\#}} \mu_{x}(f) d \varepsilon_{a}(x)-\int_{K^{\#}} \mu_{x}(f) d \mu_{a}(x) \\
& =\mu_{a}(f)-\int_{K^{\#}} \varepsilon_{x}(f) d \mu_{a}(x) \\
& =\mu_{a}(f)-\int_{K^{\#}} f(x) d \mu_{a}(x)=0
\end{aligned}
$$

where we used the fact that $\mu_{x}=\varepsilon_{x}$ for $x \in \operatorname{supp} \mu_{a}$.

Finally, it is proved in [19] that $K^{\#}$ is canonically embedded in $X$ and in this embedding we have ext $X=K^{\#} \backslash A$ and $\delta_{x}=\mu_{x}$ for $x \in K^{\#}$.

The notation introduced in this section will be used in what follows. A simplex $X$ constructed in the described way will be called a Stacey simplex associated to the pair $(K, A)$.

3. Main results. We study mainly the following questions: When any continuous (bounded Baire-one) function on $X$ can be modified to an affine Baire-one function coinciding with the original one on ext $X$; and when any bounded continuous (Baire-one) function on ext $X$ can be extended to an affine Baire-one function on $X$ ? These properties are characterized, within Stacey simplices, by the properties of the pair $(K, A)$ and the spaces $T_{a}$.

TheOrem 1. Let $K$ be a compact space, $A \subset K$ and $X$ be a Stacey simplex associated to the pair $(K, A)$. The following assertions are equivalent.

(1) Any point of $A$ is $G_{\delta}$ in $K$ and $T_{a}$ is $\sigma$-compact for each $a \in A$.

(2) For any $f: X \rightarrow \mathbb{R}$ continuous there is an affine Baire-one function $g: X \rightarrow \mathbb{R}$ with $\left.g\right|_{\operatorname{ext} X}=\left.f\right|_{\operatorname{ext} X}$.

Theorem 2. Let $K$ be a compact space, $A \subset K$ and $X$ be a Stacey simplex associated to the pair $(K, A)$. The following assertions are equivalent.

(1) ext $X$ is Lindelöf.

(2) $K \backslash A$ is Lindelöf, any compact subset of $A$ is countable and $T_{a}$ is $\sigma$-compact for any $a \in A$.

(3) For any bounded continuous function $f:$ ext $X \rightarrow \mathbb{R}$ there is an affine Baire-one function $g: X \rightarrow \mathbb{R}$ with $f=\left.g\right|_{\text {ext } X}$.

Moreover, if these conditions are satisfied, then each point of $A$ is $G_{\delta}$ in $K$ and ext $X$ is hereditarily Baire.

TheOREM 3. Let $K$ be a compact space, $A \subset K$ and $X$ be a Stacey simplex associated to the pair $(K, A)$. The following assertions are equivalent.

(1) ext $X$ is a Lindelöf $H$-set.

(2) $A$ is scattered, each point of $A$ is $G_{\delta}$ in $K$ and $T_{a}$ is $\sigma$-compact for each $a \in A$. 
(3) For any bounded Baire-one function $f:$ ext $X \rightarrow \mathbb{R}$ there is an affine Baire-one function $g: X \rightarrow \mathbb{R}$ with $f=\left.g\right|_{\operatorname{ext} X}$.

(4) For any bounded Baire-one function $f: X \rightarrow \mathbb{R}$ there is an affine Baire-one function $g: X \rightarrow \mathbb{R}$ with $\left.g\right|_{\operatorname{ext} X}=\left.f\right|_{\operatorname{ext} X}$.

Recall that $A$ is scattered if the family $\{\{a\}: a \in A\}$ is scattered.

The above theorems define three classes of Stacey simplices. The following theorem shows that these classes are different and do not cover all Stacey simplices. In the last section we show that by considering functions of higher Baire classes we get no more classes of Stacey simplices.

THEOREM 4. There are Choquet simplices $X_{1}, \ldots, X_{4}$ satisfying the following conditions.

- There is a continuous function $f_{1}: X_{1} \rightarrow \mathbb{R}$ such that no affine Baireone function on $X_{1}$ coincides with $f_{1}$ on ext $X_{1}$. Moreover, ext $X_{1}$ can be discrete (and uncountable).

- ext $X_{2}$ is an uncountable discrete space and, moreover,

(i) for each continuous $f: X_{2} \rightarrow \mathbb{R}$ there is an affine Baire-one function on $X_{2}$ which coincides with $f$ on ext $X_{2}$;

(ii) there is a bounded continuous function on ext $X_{2}$ which cannot be extended to an affine Baire-one function on $X_{2}$.

- ext $X_{3}$ is Lindelöf and, moreover,

(i) each bounded continuous $f:$ ext $X_{3} \rightarrow \mathbb{R}$ can be extended to an affine Baire-one function on $X_{3}$;

(ii) there is a bounded Baire-one function $f_{3}: X_{3} \rightarrow \mathbb{R}$ such that no affine Baire-one function on $X_{3}$ coincides with $f_{3}$ on ext $X_{3}$.

- ext $X_{4}$ is not $\check{C}$ ech analytic (in particular it is not Borel) but any bounded Baire-one function $f:$ ext $X_{4} \rightarrow \mathbb{R}$ can be extended to an affine Baire-one function on $X_{4}$.

All these examples are Stacey simplices. The simplex $X_{1}$ does not satisfy the equivalent conditions of Theorem $1, X_{2}$ satisfies the conditions of Theorem 1 but not those of Theorem $2, X_{3}$ satisfies the conditions of Theorem 2 but not those of Theorem 3 .

Note that $X_{1}$ and $X_{2}$ can be chosen such that ext $X_{1}$ and ext $X_{2}$ are homeomorphic. Hence we cannot add to the equivalent conditions of Theorem 1 another one which would be a topological property of ext $X$.

Further note that for $X_{3}$ assertion (i) follows from Theorem 2 and that the existence of a Choquet simplex (not from Stacey's class) with the same properties as $X_{3}$ is a known fact. Due to [17, Theorem 2] it is enough to take any metrizable simplex whose set of extreme points is not $F_{\sigma}$. 
The example $X_{4}$ strengthens [17, Example 3] where a Choquet simplex $X$ is described such that any bounded Baire-one function on ext $X$ can be extended to an affine Baire-one function but ext $X$ is not $F_{\sigma}$. However, ext $X$ is Borel. In our example ext $X_{4}$ is not even Cech analytic. (Recall that a subset of a compact space is $\check{C}$ ech analytic if it is a result of the Suslin operation applied to Borel sets.)

4. Auxiliary results. Recall that a subset $A$ of a topological space $X$ is called a zero set if $A=f^{-1}(0)$ for a continuous function $f: X \rightarrow \mathbb{R}$. By a $Z_{\sigma}$ set we will mean a countable union of zero sets. If $X$ is compact, then a subset of $X$ is a zero set if and only if it is a closed $G_{\delta}$-set.

The following lemma characterizes Baire-one functions on compact spaces. For the proof see [12, Exercise 3.A.1].

Lemma 1. Let $X$ be a compact space and $f: X \rightarrow \mathbb{R}$. Then the following assertions are equivalent.

(a) $f$ is a Baire-one function.

(b) $f^{-1}(U)$ is $F_{\sigma}$ in $X$ for each $U \subset \mathbb{R}$ open.

(c) $f^{-1}(U)$ is $Z_{\sigma}$ in $X$ for each $U \subset \mathbb{R}$ open.

The next lemma is a special case of an abstract reduction principle (see the proof of [10, §26, II, Theorem 1], cf. [9, Proposition 27]).

Lemma 2. Let $\left\{F_{n}: n \in \mathbb{N}\right\}$ be a cover of a topological space $X$ by $Z_{\sigma}$-sets. Then there is a partition $\left\{H_{n}: n \in \mathbb{N}\right\}$ of $X$ into $Z_{\sigma}$-sets such that $H_{n} \subset F_{n}$ for each $n$.

Proposition 3. Let $X$ be a compact convex subset of a locally convex space. Then the following assertions are equivalent.

(a) For any bounded Baire-one function $f: X \rightarrow \mathbb{R}$ there is an affine Baire-one function $g: X \rightarrow \mathbb{R}$ with $\left.g\right|_{\operatorname{ext} X}=\left.f\right|_{\operatorname{ext} X}$.

(b) $X$ is a simplex and the mapping $x \mapsto \delta_{x}(f)$ is Baire-one for any bounded Baire-one function on $X$.

(c) $X$ is a simplex and the mapping $x \mapsto \delta_{x}(A)$ is Baire-one for any $A \subset X$ which is simultaneously $F_{\sigma}$ and $G_{\delta}$.

(d) $X$ is a simplex and the mapping $x \mapsto \delta_{x}(U)$ is Baire-one for any open $F_{\sigma}$-set $U \subset X$.

Proof. The equivalence (a) $\Leftrightarrow(\mathrm{b})$ is proved in [17, Corollary 1]. The implication $(\mathrm{b}) \Rightarrow(\mathrm{d})$ is trivial as $\chi_{U}$ is Baire-one whenever $U$ is open $F_{\sigma}$.

(d) $\Rightarrow\left(\right.$ c). Let $A \subset X$ be simultaneously $F_{\sigma}$ and $G_{\delta}$. It follows by Lemma 1 that $\chi_{A}$ is Baire-one and hence both $A$ and $X \backslash A$ are $Z_{\sigma}$. Then $A=\bigcup_{n \in \mathbb{N}} A_{n}$ where each $A_{n}$ is a closed $G_{\delta}$ and $A_{n} \subset A_{n+1}$ for each $n$. 
Hence for any constant $c$ we have

$$
\begin{aligned}
\left\{x \in X: \delta_{x}(A)>c\right\} & =\bigcup_{n \in \mathbb{N}}\left\{x \in X: \delta_{x}\left(A_{n}\right)>c\right\} \\
& =\bigcup_{n \in \mathbb{N}}\left\{x \in X: \delta_{x}\left(X \backslash A_{n}\right)<1-c\right\} .
\end{aligned}
$$

This set is clearly $F_{\sigma}$. The same can be done for $X \backslash A$ in place of $A$ and hence

$$
\left\{x \in X: \delta_{x}(A)<c\right\}=\left\{x \in X: \delta_{x}(X \backslash A)>1-c\right\}
$$

is $F_{\sigma}$ as well. It follows by Lemma 1 that $x \mapsto \delta_{x}(A)$ is Baire-one.

(c) $\Rightarrow(\mathrm{b})$. Let $f: X \rightarrow \mathbb{R}$ be a bounded Baire-one function. Fix $n \in \mathbb{N}$. For $k \in \mathbb{Z}$ put $U_{n, k}=f^{-1}\left(\left(\frac{k-1}{n}, \frac{k+1}{n}\right)\right)$. Then each $U_{n, k}$ is a $Z_{\sigma}$-subset of $X$ (by Lemma 1), the sets $U_{n, k}, k \in \mathbb{Z}$, cover $X$ and $U_{n, k} \neq \emptyset$ only for finitely many $k$ 's. By Lemma 2 there are $Z_{\sigma}$-sets $V_{n, k} \subset U_{n, k}$ which are pairwise disjoint and cover $X$. Set $h_{n}=\sum_{k \in \mathbb{Z}}(k / n) \chi_{V_{n, k}}$.

Then clearly the sequence $h_{n}$ converges uniformly to $f$.

Further, the function $x \mapsto \delta_{x}\left(h_{n}\right)$ is Baire-one. Indeed, we have

$$
\delta_{x}\left(h_{n}\right)=\sum_{k \in \mathbb{Z}} \frac{k}{n} \delta_{x}\left(V_{n, k}\right),
$$

$V_{n, k}$ is simultaneously $F_{\sigma}$ and $G_{\delta}$, and $V_{n, k}$ is nonempty only for a finite number of $k$ 's.

Finally, the function $x \mapsto \delta_{x}(f)$ is a uniform limit of functions from the previous paragraph and hence it is also a Baire-one function.

Lemma 4. Let $K$ be a compact space and $A \subset K$ an arbitrary subset. If each compact subset of $A$ is $G_{\delta}$ in $K$, then $K \backslash A$ is Lindelöf.

Proof. Let $\left\{U_{a}: a \in I\right\}$ be a covering of $K \backslash A$ consisting of relatively open sets. We are going to show that there is a countable subcover.

If there is a finite subcover, we are done. Otherwise we set $F_{a}=$ $(K \backslash A) \backslash U_{a}$ for $a \in I$ and remark that the family $\left\{F_{a}: a \in I\right\}$ has the finite intersection property and hence $F=\bigcap_{a \in I} \bar{F}_{a} \neq \emptyset$ where the closures are taken in $K$. But each $F_{a}$ is relatively closed in $K \backslash A$ and $\bigcap_{a \in I} F_{a}=\emptyset$ (as the $U_{a}$ 's cover $K \backslash A$ ). Therefore $F \subset A$ and hence $F$ is $G_{\delta}$ in $K$.

Fix a sequence $G_{n}$ of open subsets of $K$ with $F=\bigcap_{n \in \mathbb{N}} G_{n}$. For each $n \in \mathbb{N}$ there is a finite set $J_{n} \subset I$ with $\bigcap_{a \in J_{n}} F_{a} \subset G_{n}$. If we set $J=$ $\bigcup_{n \in \mathbb{N}} J_{n}$ then $J$ is a countable subset of $I$ satisfying $\bigcap_{a \in J} F_{a}=\emptyset$ and hence $\bigcup_{a \in J} U_{a}=K \backslash A$.

LEMMA 5. Any compact scattered space for which each point is $G_{\delta}$ (i.e., which is first countable) is countable.

Proof. Recall that the Cantor-Bendixson derivative is defined as follows: $L^{(0)}=L, L^{(\alpha+1)}$ is the set of all accumulation points of $L^{(\alpha)}$, and $L^{(\lambda)}=$ 
$\bigcap_{\alpha<\lambda} L^{(\alpha)}$ if $\lambda$ is a limit ordinal. The Cantor-Bendixson height of $L$ is the minimal $\alpha$ such that $L^{(\alpha)}=\emptyset$.

Suppose that there is an uncountable scattered first countable compact space. Let $L$ be such a space with the minimal possible Cantor-Bendixson height and $\alpha$ be this height. Then clearly $\alpha$ is not limit and hence $\alpha=\beta+1$ for some ordinal $\beta$. Then $L^{(\beta)}$ is finite and hence $G_{\delta}$. So its complement is $F_{\sigma}$, i.e.

$$
L \backslash L^{(\beta)}=\bigcup_{n \in \mathbb{N}} L_{n}
$$

with each $L_{n}$ compact. As $L$ is uncountable, there is $n \in \mathbb{N}$ such that $L_{n}$ is uncountable. Then $L_{n}$ is a scattered uncountable first countable compact space with Cantor-Bendixson height less than $\alpha$ (as clearly $\left.\left(L_{n}\right)^{(\beta)}=\emptyset\right)$, which contradicts the minimality of $\alpha$.

Lemma 6. Let $K$ be a compact space and $C \subset K$ a countable scattered set such that each point of $C$ is a $G_{\delta}$-point of $K$. Then $C$ is $G_{\delta}$ in $K$.

Proof. As $C$ is countable and scattered, there are a well-ordering $\left\{c_{\alpha}: \alpha<\xi\right\}$ of $C$ (where $\xi$ is a countable ordinal) and open $F_{\sigma}$-sets $\left\{U_{\alpha}: \alpha<\xi\right\}$ with $c_{\alpha} \in U_{\alpha} \backslash \bigcup_{\beta<\alpha} U_{\beta}$.

Then $U=\bigcup_{\alpha<\xi} U_{\alpha}$ is an open $F_{\sigma}$-set. Further set $V_{\alpha}=U_{\alpha} \backslash \bigcup_{\beta<\alpha} U_{\beta}$ for $\alpha<\xi$. Then each $V_{\alpha}$ is simultaneously $F_{\sigma}$ and $G_{\delta}$ and $U=\bigcup_{\alpha<\xi} V_{\alpha}$. As each $c_{\alpha}$ is a $G_{\delta}$-point, each $V_{\alpha} \backslash\left\{c_{\alpha}\right\}$ is $F_{\sigma}$. Therefore

is $G_{\delta}$.

$$
C=U \backslash \bigcup_{\alpha<\xi}\left(V_{\alpha} \backslash\left\{c_{\alpha}\right\}\right)
$$

Lemma 7. Let $X$ be a topological space with countable tightness which is not scattered. Then there is a nonempty countable set $C \subset X$ without isolated points.

Proof. As $X$ is not scattered, there is a nonempty subset $B \subset X$ without isolated points. Choose $b \in B$, set $C_{0}=\{b\}$ and construct by induction countable sets $C_{n}, n=1,2, \ldots$, in the following manner.

Let $C_{n}^{\prime} \subset B \backslash C_{n-1}$ be a countable set such that $\overline{C_{n}^{\prime}}$ contains all isolated points of $C_{n-1}$ (such a set exists as $C_{n-1}$ is countable, $B$ has countable tightness and has no isolated points) and $C_{n}=C_{n-1} \cup C_{n}^{\prime}$. Then $C=$ $\bigcup_{n=0}^{\infty} C_{n}$ is a nonempty countable set without isolated points.

Proposition 8. Let $X$ be a Choquet simplex and $f: \overline{\operatorname{ext} X} \rightarrow \mathbb{R}$ be a bounded Baire-one function. Define the function $g: X \rightarrow \mathbb{R}$ by the formula

$$
g(x)=\delta_{x}(f), \quad x \in X
$$

Then $g$ satisfies the barycentric formula. Moreover, $g$ is Baire-one if (and only if ) $\left.g\right|_{\operatorname{ext} X}$ is Baire-one. 
Proof. Let us first show that $g$ satisfies the barycentric formula. This follows from the proof of [11, Proposition 6.1]. However, the cited result is stated for metrizable simplices, so we give the proof.

First suppose that $f$ is a convex continuous function defined on $X$. Then by [11, Lemma 2.3] we have

$$
\begin{array}{r}
g(x)=\inf \{h(x): h \text { is continuous and affine on } X, h \geq f \text { on } X\}, \\
x \in X .
\end{array}
$$

Let $\mu \in P(X)$ be arbitrary. Then

$$
\mu(g)=\inf \{\mu(h): h \text { is continuous and affine on } X, h \geq f \text { on } X\} .
$$

This follows from [6, Theorem 9.11] as the set

$$
\{h \text { affine continuous on } X: h \geq f\}
$$

is downward directed (i.e. for any $h_{1}, h_{2}$ in this set there is $h$ in the set such that $\left.h \leq \min \left\{h_{1}, h_{2}\right\}\right)$ due to Edwards's separation theorem [4, Theorem 3].

Further, for any $h$ affine continuous on $X$ we have $\mu(h)=h(r(\mu))$, hence $\mu(g)=\inf \{h(r(\mu)): h$ is continuous and affine on $X, h \geq f$ on $X\}=g(r(\mu))$ by $(*)$. Thus $g$ satisfies the barycentric formula if $f$ is any continuous convex function on $X$.

Notice that the family

$\left\{f: X \rightarrow \mathbb{R}\right.$ universally measurable $\left.:(\forall \mu \in P(X))\left(\int \delta_{x}(f) d \mu(x)=\delta_{r(\mu)}(f)\right)\right\}$

is a linear space closed under pointwise limits of bounded sequences (due to the Lebesgue dominated convergence theorem). We have already shown that it contains all convex continuous functions. As differences of convex continuous functions form a lattice containing the constants and separating points of $X$, they are, due to the Stone-Weierstrass theorem, uniformly dense in $\mathcal{C}(X)$. Hence our family contains all continuous functions and thus also all bounded Baire-one functions.

If $f$ is a bounded Baire-one function on $\overline{\operatorname{ext} X}$, it can be extended to a bounded Baire-one function on $X$ (see e.g. [9, Theorem 13 or Remark 17]). As any maximal measure is supported by $\overline{\operatorname{ext} X}$, we get the first part.

The second part now follows immediately from [16, Theorem 3.3].

\section{Proofs of the main results}

Proof of Theorem 1. (2) $\Rightarrow(1)$. Let $a \in A$ be arbitrary. Then supp $\mu_{a}$ is a compact subset of $\{a\}^{\#}$ not containing $(a, a)$. Choose a relatively open set $U \subset\{a\}^{\#}$ containing $(a, a)$ such that $\bar{U} \cap \operatorname{supp} \mu_{a}=\emptyset$. By Urysohn's lemma there is a continuous function $f_{0}:\{a\}^{\#} \rightarrow[0,1]$ with $\left.f_{0}\right|_{\operatorname{supp} \mu_{a}}=1$ 
and $\left.f_{0}\right|_{U}=0$. Define $f_{1}: K^{\#} \rightarrow[0,1]$ by

$$
f_{1}(x)= \begin{cases}f_{0}(x), & x \in\{a\}^{\#}, \\ 0, & x \in K^{\#} \backslash\{a\}^{\#} .\end{cases}
$$

Then $f_{1}$ is continuous on $K^{\#}$ and hence it can be (due to Tietze's theorem) extended to a continuous function $f: X \rightarrow[0,1]$. Let $g$ be a function satisfying the barycentric formula such that $g$ coincides with $f$ on $\operatorname{ext} X$. Then

$$
g(x)= \begin{cases}1, & x=(a, a), \\ 0, & x \in K^{\#} \backslash\{a\}^{\#}, \\ 0, & x \in U \backslash\{a, a\} .\end{cases}
$$

If $g$ is Baire-one, then $(a, a)$ is a $G_{\delta}$-point of $K^{\#}$ by Lemma 1 . Hence clearly $a$ is a $G_{\delta}$-point of $K$ and $T_{a}$ is $\sigma$-compact.

$(1) \Rightarrow(2)$. Let $f: X \rightarrow \mathbb{R}$ be continuous. Put $g(x)=\delta_{x}(f)$. By Proposition 8 it is enough to check that $g$ is Baire-one on $K^{\#}$. Note that

$$
g(x)= \begin{cases}\mu_{x}(f), & x \in A \\ f(x), & \text { otherwise. }\end{cases}
$$

It follows easily from the continuity of $f$ that

$$
\left\{x \in A:\left|f(x)-\mu_{x}(f)\right|>c\right\}
$$

is finite for each $c>0$. Hence the sets

$$
\left\{x \in K^{\#}: f(x)-g(x)>c\right\} \quad \text { and } \quad\left\{x \in K^{\#}: f(x)-g(x)<c\right\}
$$

are $F_{\sigma}$ for each $c \in \mathbb{R}$. Indeed, the first set is finite for $c>0$ and countable for $c=0$. For $c<0$ it is the complement of a finite subset of $A$, hence it is also an $F_{\sigma}$-set (as it follows from (1) that any point of $A$ is $G_{\delta}$ in $K^{\#}$ ). The proof for the second set is analogous.

Hence $\left.(f-g)\right|_{K^{\#}}$ is Baire-one by Lemma 1. Finally, $\left.g\right|_{K^{\#}}=\left.f\right|_{K^{\#}}-$ $\left.(f-g)\right|_{K^{\#}}$ is Baire-one.

Proof of Theorem 2. (1) $\Rightarrow(2)$. Suppose ext $X$ is Lindelöf. Then $K \backslash A$ is also Lindelöf, being a closed subset of ext $X$. Further, if $F \subset A$ is compact, then $F^{\#} \backslash F$ is closed in ext $X$ and hence it is also Lindelöf. Since $\{a\} \times T_{a}$, $a \in F$, is a partition of $F^{\#} \backslash F$ into nonempty open sets, $F$ must be countable. Finally, for each $a \in A$ the set $\{a\} \times T_{a}$ is a closed subset of ext $X$ and hence it is Lindelöf. As $T_{a}$ is locally compact and Lindelöf, it is $\sigma$-compact.

$(2) \Rightarrow(1)$. Let $\mathcal{U}$ be a family of basic open sets in $K^{\#}$ covering ext $X$. As $K \backslash A$ is Lindelöf, there is a countable subfamily $\mathcal{U}_{1} \subset \mathcal{U}$ covering $K \backslash A$. We can suppose that any element of $\mathcal{U}_{1}$ intersects $K \backslash A$. In this case any $U \in \mathcal{U}_{1}$ is of the form

$$
U=\left(G_{U} \backslash\left\{k_{U}\right\}\right)^{\#} \cup\left\{k_{U}\right\} \times\left(\left\{k_{U}\right\} \cup T_{k_{U}} \backslash H_{U}\right),
$$


where $k_{U} \in K, G_{U}$ is an open neighborhood of $k_{U}$ in $K$ and $H_{U} \subset T_{k_{U}}$ is compact. Then $G_{U}, U \in \mathcal{U}_{1}$, cover $K \backslash A$ and hence $F=K \backslash \bigcup\left\{G_{U}: U \in \mathcal{U}_{1}\right\}$ is a compact subset of $A$. Thus $F$ is countable. Therefore $\mathcal{U}_{1}$ covers ext $X$ except for the $\sigma$-compact set $\left(F^{\#} \backslash F\right) \cup \bigcup_{U \in \mathcal{U}_{1}}\left\{k_{U}\right\} \times H_{U}$. It follows that we can add further countably many elements of $\mathcal{U}$ to $\mathcal{U}_{1}$ to get a countable subcover of ext $X$.

$(1) \Rightarrow(3)$. This implication holds for general simplices and is proved in [8].

$(3) \Rightarrow\left(2 \&\right.$ each point of $A$ is $G_{\delta}$ in $\left.K\right)$. It follows from Theorem 1 that each point of $A$ is $G_{\delta}$ in $K$ and $T_{a}$ is $\sigma$-compact for each $a \in A$.

Further, if $F \subset A$ is compact and $B \subset F$, then $B^{\#} \backslash B$ is a clopen subset of ext $X$. Let $f$ be the characteristic function of $B^{\#} \backslash B$. By our assumption it can be extended to an affine Baire-one function $g: X \rightarrow \mathbb{R}$. Then necessarily

$$
g((k, k))= \begin{cases}1, & k \in B, \\ 0, & k \in K \backslash B .\end{cases}
$$

So $B$ is $G_{\delta}$ in $K$ by Lemma 1 .

Hence, in particular, any compact subset of $A$ is $G_{\delta}$ in $K$ and therefore $K \backslash A$ is Lindelöf by Lemma 4 .

Finally, if $F \subset A$ is compact, then any subset of $F$ is $G_{\delta}$ in $F$. We claim that $F$ is necessarily countable.

Let us first show that $F$ is scattered. If not, we get by Lemma 7 a nonempty countable $C \subset F$ without isolated points (note that $F$ is first countable and thus has countable tightness). Hence $C$ is meager in itself. However, $C$ is $G_{\delta}$ in $F$, so it is Cech complete and thus a Baire space. This is a contradiction.

Then, by Lemma $5, F$ is countable.

$(2) \Rightarrow$ (ext $X$ is hereditarily Baire). It is clearly enough to show that $K \backslash A$ is hereditarily Baire. Suppose not. Then there is $H \subset K \backslash A$ relatively closed which is meager in itself. Let $F$ be the closure of $H$ in $K$. Then $F \backslash H=F \cap A$ has no isolated points and contains a dense $G_{\delta}$-subset of $F$. (Indeed, let $H_{n}, n \in \mathbb{N}$, be relatively closed nowhere dense subsets of $H$ covering $H$. Then $\bar{H}_{n}$ is nowhere dense in $F$ and $F \backslash \bigcup_{n \in \mathbb{N}} \bar{H}_{n}$ is a dense $G_{\delta}$-subset of $F$ contained in $F \backslash H$.) Hence $A$ contains a nonempty Čech complete subset without isolated points. But any such set contains an uncountable compact subset, a contradiction.

Proof of Theorem 3. (1) $\Rightarrow(2)$. It follows from Theorem 2 that each point of $A$ is $G_{\delta}$ in $K, T_{a}$ is $\sigma$-compact for each $a \in A$ and any compact subset of $A$ is countable. As ext $X$ is an $H$-set and $K \backslash A$ is closed in ext $X$, we see that $K \backslash A$ is an $H$-set in $K$, hence $A$ is also an $H$-set. Suppose $A$ is not scattered. Then there is a nonempty relatively closed subset $F \subset A$ without isolated points. Then $F$ is also an $H$-set and hence contains a dense locally compact 
subset. (Note that $F$ is dense in the compact space $\bar{F}$ and hence, by the very definition of an $H$-set, there is a dense relatively open subset of $\bar{F}$ contained in $F$. This set is clearly locally compact.) But any nonempty locally compact subset without isolated points contains an uncountable compact subset. This is a contradiction showing that $A$ is scattered.

$(2) \Rightarrow(1)$. If $A$ is scattered, then ext $X$ is clearly an $H$-set. If each point of $A$ is $G_{\delta}$ in $K$, then each compact subset of $A$ is countable by Lemma 5 and each countable subset of $A$ is $G_{\delta}$ in $K$ by Lemma 6 . Hence, any compact subset of $A$ is $G_{\delta}$ in $K$ and so $K \backslash A$ is Lindelöf by Lemma 4 . Finally, ext $X$ is Lindelöf by Theorem 2 .

$(4) \Rightarrow(2)$. It follows from Theorem 1 that each point of $A$ is $G_{\delta}$ in $K$ and $T_{a}$ is $\sigma$-compact for each $a \in A$.

Further, let $C \subset A$ be countable. Then $\chi_{C \# \backslash C}$ is a bounded Baire-one function on $K^{\#}$. Let $f$ be a bounded Baire-one function on $X$ extending $\chi_{C \# \backslash C}$ (such a function exists due to [9, Theorem 13]). Let $g$ be an affine Baire-one function on $X$ coinciding with $f$ on ext $X$. Then $g((k, k))=\chi_{C}(k)$ for each $k \in K$. Hence $C$ is $G_{\delta}$ in $K$ by Lemma 1 .

We have shown that each countable subset of $A$ is $G_{\delta}$ in $K$. We will deduce that $A$ is scattered. Suppose it is not. As $A$ is first countable, we get by Lemma 7 a nonempty countable set $C \subset A$ without isolated points. Then $C$ is meager in itself and simultaneously $G_{\delta}$ in $K$ and hence Cech complete, a contradiction.

$(2) \Rightarrow(4)$. By Proposition 3 it is enough to show that $x \mapsto \delta_{x}(U)$ is Baireone whenever $U \subset X$ is open $F_{\sigma}$. By Proposition 8 it suffices to prove that this mapping is Baire-one on $K^{\#}$.

So let $U \subset K^{\#}$ be open $F_{\sigma}$. Then

$$
\begin{aligned}
U= & \bigcup_{n \in \mathbb{N}}\left(\left\{l_{n}\right\} \times V_{n}\right) \\
& \cup \bigcup_{n \in \mathbb{N}}\left(U_{n} \backslash\left\{k_{n}\right\}\right) \# \cup\left\{k_{n}\right\} \times\left(\left\{k_{n}\right\} \cup T_{k_{n}} \backslash H_{n}\right)
\end{aligned}
$$

where $l_{n} \in A, V_{n}$ is an open $\sigma$-compact subset of $T_{l_{n}}, k_{n} \in K, U_{n}$ is an open $F_{\sigma}$ subset of $K$, and $H_{n}$ is a compact subset of $T_{k_{n}}$ which is $G_{\delta}$ in $\left\{k_{n}\right\}^{\#}$ for $n \in \mathbb{N}$.

The set $U$ is thus written as $W_{1} \cup W_{2}$. We claim that we can without loss of generality suppose $W_{1} \cap W_{2}=\emptyset$. To see this it is enough to show that, without loss of generality, $\left\{l_{n}: n \in \mathbb{N}\right\} \cap \bigcup_{n \in \mathbb{N}} U_{n}=\emptyset$. Suppose that, for some $m, n \in \mathbb{N}$ we have $l_{m} \in U_{n}$. If $l_{m} \neq k_{n}$, then $\left\{l_{m}\right\} \times V_{m} \subset W_{2}$ and hence it can be omitted from $W_{1}$. If $l_{m}=k_{n}$, we can omit $\left\{l_{m}\right\} \times V_{m}$ and replace $H_{n}$ by $H_{n} \backslash V_{m}$.

Therefore, as the sum of two Baire-one functions is Baire-one, it is enough to consider two cases: 
(i) $U=\bigcup_{n \in \mathbb{N}}\left(\left\{l_{n}\right\} \times V_{n}\right)$. Then

$$
\delta_{x}(U)= \begin{cases}1, & x \in \bigcup_{n \in \mathbb{N}}\left(\left\{l_{n}\right\} \times V_{n}\right), \\ \mu_{x}\left(\left\{l_{n}\right\} \times V_{n}\right), & x=l_{n}, n \in \mathbb{N}, \\ 0, & \text { otherwise. }\end{cases}
$$

The set $\bigcup_{n \in \mathbb{N}}\left(\left\{l_{n}\right\} \times V_{n}\right)$ is open $F_{\sigma}$ in $K^{\#}$, each subset of $\left\{l_{n}: n \in \mathbb{N}\right\}$ is simultaneously $F_{\sigma}$ (being countable) and $G_{\delta}$ (by Lemma 6 ) in $K^{\#}$. (We use the fact that each point of $A$ is $G_{\delta}$ in $K^{\#}$. This follows easily from the assumptions of (2).) Now it is clear that

$$
\left\{x \in K^{\#}: \delta_{x}(U)>c\right\} \text { and }\left\{x \in K^{\#}: \delta_{x}(U)<c\right\}
$$

are $F_{\sigma}$ in $K^{\#}$ for each $c \in \mathbb{R}$. It follows by Lemma 1 that the function $x \mapsto \delta_{x}(U)$ is Baire-one on $K^{\#}$.

(ii) $U=\bigcup_{n \in \mathbb{N}}\left(U_{n} \backslash\left\{k_{n}\right\}\right)^{\#} \cup\left\{k_{n}\right\} \times\left(\left\{k_{n}\right\} \cup T_{k_{n}} \backslash H_{n}\right)$. Then we have $U=G^{\#} \backslash \bigcup_{n \in \mathbb{N}}\left\{h_{n}\right\} \times H_{n}$ for an open $F_{\sigma^{-}}$set $G \subset K$ and suitable $h_{n} \in A$ and $H_{n} \subset T_{h_{n}}$ closed $G_{\delta}$ in $\left\{h_{n}\right\}^{\#}$. Then

$$
\delta_{x}(U)= \begin{cases}1, & \left.x \in U \backslash\left\{h_{n}: n \in \mathbb{N}\right\}\right), \\ 1-\mu_{h_{n}}\left(\left\{h_{n}\right\} \times H_{n}\right), & x=h_{n}, n \in \mathbb{N}, \\ 0, & \text { otherwise. }\end{cases}
$$

As $U$ is open $F_{\sigma}$ and each subset of $\left\{h_{n}: n \in \mathbb{N}\right\}$ is simultaneously $F_{\sigma}$ and $G_{\delta}$ in $K^{\#}$ (Lemma 6), it easily follows from Lemma 1 that $x \mapsto \delta_{x}(U)$ is Baire-one on $K^{\#}$.

The implication $(3) \Rightarrow(4)$ is trivial.

$(2) \Rightarrow(3)$. Let $(2)$ hold and $f: \operatorname{ext} X \rightarrow \mathbb{R}$ be a bounded Baire-one function. By the already proved implication $(2) \Rightarrow(1)$ we know that $\operatorname{ext} X$ is Lindelöf. Therefore there is, due to [9, Theorem 30], a bounded Baire-one function $\tilde{f}: X \rightarrow \mathbb{R}$ extending $X$. So we can conclude by using the already proved implication $(2) \Rightarrow(4)$.

Proof of Theorem 4. All the examples will be Stacey simplices associated to suitable pairs $(K, A)$ such that $T_{a}$ is a two-point set for each $a \in A$. In this case each $T_{a}$ is automatically $\sigma$-compact. For $\mu_{a}$ we can take, for example, $\frac{1}{2}\left(\varepsilon_{\left(a, t_{1}\right)}+\varepsilon_{\left(a, t_{2}\right)}\right)$ where $\left\{t_{1}, t_{2}\right\}=T_{a}$.

- To define $X_{1}$ take $K=A$ where $K$ is any compact space which is not first countable. Then ext $X_{1}$ is uncountable discrete and some point of $A$ is not a $G_{\delta}$-point in $K$. The required properties follow from Theorem 1. As concrete examples, we can take $K$ to be the ordinal interval $[0, \alpha]$ with $\alpha \geq \omega_{1}$ or the one-point compactification of an uncountable discrete space.

- To construct $X_{2}$ take $K=A$ where $K$ is an uncountable first countable compact space. Then ext $X_{2}$ is uncountable and discrete. Property (i) follows from Theorem 1, property (ii) from Theorem 2. As a concrete example we can take $K=[0,1]$. 
- To define $X_{3}$ we can choose $K=[0,1]$ and $A$ to be an uncountable subset of $[0,1]$ which contains no uncountable closed set. This is the case, for example, if $A$ is an uncountable perfectly meager set [10, III, $\S 40$, II and III] or a Bernstein set [13, Theorem 5.3]. The properties then follow from Theorems 2 and 3 (note that an uncoutable subset of the real line cannot be scattered).

- To find $X_{4}$ set $K=\left[0, \omega_{1}\right]$ and let $A \subset\left[0, \omega_{1}\right)$ be a stationary and costationary set (i.e. both $A$ and its complement intersect any closed unbounded set in $\left.\left[0, \omega_{1}\right)\right)$. Such a set exists by [5] and it follows from [7, Lemma 7$]$ that it is not Cech analytic. Hence ext $X_{4}$ is not Cech analytic. Finally, $K$ and $A$ satisfy (2) of Theorem (3). This completes the proof.

6. Functions of higher Baire class. It is natural to ask what happens for functions of a higher Baire class. Recall that functions of Baire class $\alpha$ are pointwise limits of sequences of functions of Baire class less than $\alpha$. Baire functions are functions of Baire class $\alpha$ for some $\alpha$ (where it is enough to consider $\left.\alpha<\omega_{1}\right)$.

The situation for higher classes may be different from the first class case. While affine Baire-one functions satisfy the barycentric formula, affine Baire-two functions need not.

For example, if $X=P([0,1])$, then the function assigning to each $\mu$ the number $\mu_{d}([0,1])$ where $\mu_{d}$ is the discrete part of $\mu$ is an affine Baire-two function not satisfying the barycentric formula. For the sake of completeness we give the proof of this folklore fact.

The function is clearly affine. To see that it does not satisfy the barycentric formula, consider the measure $\Lambda$ on $X$ defined by $\Lambda(A)=\lambda(\{x \in[0,1]$ : $\left.\left.\varepsilon_{x} \in A\right\}\right)$, where $\lambda$ denotes the Lebesgue measure. It is easy to check that the barycenter of $\Lambda$ is $\lambda$. Further, $\lambda_{d}([0,1])=0$, while $\int_{X} \mu_{d}([0,1]) d \Lambda(\mu)=1$.

It remains to show that our function is Baire-two. For $n \in \mathbb{N}$ define

$$
f_{n}(\mu)=\mu(\{x \in[0,1]: \mu(\{x\}) \geq 1 / n\}), \quad \mu \in X .
$$

Then $\mu_{d}([0,1])=\lim _{n \rightarrow \infty} f_{n}(\mu)$ for each $\mu \in X$. It suffices to show that each $f_{n}$ is Baire-one. As $X$ is metrizable, it is enough to show that $f_{n}$ is upper semicontinuous, i.e. $\left\{\mu \in X: f_{n}(\mu)<c\right\}$ is open for each $c>0$.

Let $\mu \in X$ be such that $f_{n}(\mu)<c$. Denote by $H$ the set of all $x \in[0,1]$ with $\mu(\{x\}) \geq 1 / n$. Then $H$ is finite and hence closed. Let $d$ be the maximal measure of a singleton in $[0,1] \backslash H$. Then $d<1 / n$. Chose $\varepsilon>0$ such that $\varepsilon<\frac{1}{3}(1 / n-d)$ and $\varepsilon<\frac{1}{2}\left(c-f_{n}(\mu)\right)$.

There is a compact set $K \subset[0,1] \backslash H$ such that $\mu(K)>\mu([0,1] \backslash H)-\varepsilon=$ $1-f_{n}(\mu)-\varepsilon$. As each singleton in $K$ has measure less than $d+\varepsilon$, we can find open sets $V_{1}, \ldots, V_{k}$ disjoint from $H$ and covering $K$ such that $\mu\left(V_{i}\right)<d+\varepsilon$ for $i=1, \ldots, k$. Set $W_{j}=V_{j} \backslash \bigcup_{i<j} V_{i}$ for $j=1, \ldots, k$. Choose compact 
subsets $F_{j} \subset W_{j}$ such that $\sum_{j=1}^{k} \mu\left(F_{j}\right)>\mu([0,1] \backslash H)-\varepsilon$. Finally, choose pairwise disjoint open sets $U_{0}, U_{1}, \ldots, U_{k}$ such that $H \subset U_{0}$ and $F_{j} \subset U_{j} \subset V_{j}$ for each $j=1, \ldots, k$. Then the set

$$
\left\{\nu \in X: \nu\left(U_{j}\right)>\mu\left(U_{j}\right)-\frac{\varepsilon}{k+1} \text { for } j=0, \ldots, k\right\}
$$

is an open subset of $X$ (see e.g. [20, Theorem 8.1]) containing $\mu$. If $\nu$ belongs to this set, it can be easily calculated that $\nu\left(U_{i}\right)<1 / n$ for $i=1, \ldots, n$, and $\nu\left([0,1] \backslash \bigcup_{i=1}^{n} U_{i}\right)<c$. Therefore $f_{n}(\nu)<c$. This completes the proof.

However, if we consider only functions satisfying the barycentric formula, we obtain within Stacey simplices the following results.

TheORem 5. Let $X$ be a Stacey simplex. If for each continuous function $f$ on $X$ there is a Baire function satisfying the barycentric formula and coinciding with $f$ on ext $X$, then the equivalent assertions of Theorem 1 hold. Moreover, in this case the following hold.

(i) For each bounded Baire-one function $f$ on $X$ there is a Baire-two function satisfying the barycentric formula and coinciding with $f$ on ext $X$.

(ii) If $\alpha \geq 2$ and $f$ is a bounded function of Baire class $\alpha$ on $X$ then there is a function of the same Baire class $\alpha$ satisfying the barycentric formula and coinciding with $f$ on $\operatorname{ext} X$.

Proof. The validity of assertion (1) of Theorem 1 can be proved in the same way as $(2) \Rightarrow(1)$ of Theorem 1 if we notice that the characteristic function of a singleton is a Baire function if and only if the corresponding point is $G_{\delta}$.

Let us further show that assertion (1) of Theorem 1 implies (i) and (ii). If $f$ is a bounded Baire function on $X$, then the function $g(x)=\delta_{x}(f)$ satisfies the barycentric formula (this follows easily from Proposition 8 ). Due to $[16$, Theorem 3.3] it is enough to prove that $f-g$ is Baire-two on $K^{\#}$. But $\left\{x \in K^{\#}: f(x)-g(x) \neq 0\right\}$ is a countable subset of $A$ (by the proof of $(1) \Rightarrow(2)$ in Theorem 1 this is true if $f$ is continuous, hence it clearly holds for all Baire functions). Let us enumerate the elements of this set as $\left\{a_{n}: n \in \mathbb{N}\right\}$. As each $a_{n}$ is $G_{\delta}$ in $K^{\#}$, the function $\chi_{\left\{a_{1}, \ldots, a_{n}\right\}} \cdot(f-g)$ is, for each $n \in \mathbb{N}$, a Baire-one function by Lemma 1 . Moreover, this sequence converges pontwise to $f-g$, therefore $f-g$ is Baire-two. This completes the proof.

Theorem 6. Let $X$ be a Stacey simplex. If for each bounded continuous function $f: \operatorname{ext} X \rightarrow \mathbb{R}$ there is a Baire function satisfying the barycentric formula and extending $f$, then the equivalent assertions of Theorem 2 hold. Moreover, in this case the following hold. 
(i) Any bounded Baire-one function on ext $X$ can be extended to a Bairetwo function satisfying the barycentric formula.

(ii) If $\alpha \geq 2$, then any bounded function of Baire class $\alpha$ on ext $X$ can be extended to a function of the same Baire class $\alpha$ satisfying the barycentric formula.

Proof. Let us first show that under our assumptions assertion (2) of Theorem 2 holds. We will proceed similarly to the proof of $(3) \Rightarrow(2)$ in Theorem 2. By Theorem 5 we know that each point of $A$ is $G_{\delta}$ in $K$ and that $T_{a}$ is $\sigma$-compact for each $a \in A$. Let $F \subset A$ be compact. Then, as in the proof of Theorem $2, \chi_{B}$ is a Baire function on $K$ for each $B \subset F$. It follows that $F$ is $G_{\delta}$ in $K$. Thus $K \backslash A$ is Lindelöf by Lemma 4 . Further, each subset of $F$ is Borel, so $F$ is scattered. (If $F$ is not scattered, it is easy to construct a closed subset $C \subset F$ and a continuous surjection $\varphi: C \rightarrow\{0,1\}^{\mathbb{N}}$ and hence $C$ contains a non-Borel set by $[14$, Lemma 2].) As $F$ is first countable, $F$ is countable by Lemma 5 .

Further, let us show that assertion (2) of Theorem 2 implies (i) and (ii). Let $f:$ ext $X \rightarrow \mathbb{R}$ be a bounded Baire function of class $\alpha$. Extend $f$ to a function $g: K^{\#} \rightarrow \mathbb{R}$ by setting $g((a, a))=\mu_{a}(f)$. We claim that $g$ is of Baire class $\max \{\alpha, 2\}$.

Let us show it first for $\alpha=2$. We remark that there is a $\sigma$-compact set $H \subset A$ such that $\left.g\right|_{\left(K_{\#}^{\#} \backslash H\right)}$ is Baire-two. Indeed, if $f$ is continuous, then $g$ is clearly continuous at each point of $K^{\#} \backslash K$ and it is easy to check that it is also continuous at each point of $K \backslash A$. Further, the set of continuity points is $G_{\delta}$, hence there is $H \subset A \sigma$-compact with $\left.g\right|_{K_{\#}^{\#} \backslash H}$ continuous. Now the claim easily follows.

As $H$ is countable and each point of $H$ is $G_{\delta}$ in $K^{\#}$, the function $\chi_{H} \cdot g$ is Baire-two on $K^{\#}$ (this can be proved by the method used in the proof of Theorem 5 above). Further, the function $\chi_{K \# \backslash H} \cdot g$ is Baire-two as well.

We already know that $g$ is Baire-two on $K^{\#} \backslash H$. Hence there is a sequence $\widetilde{h}_{n}$ of Baire-one functions on $K^{\#} \backslash H$ converging to $g$. The set $K^{\#} \backslash H$ is simultaneously $G_{\delta}$ and $F_{\sigma \delta}$ in $K^{\#}$. Thus it is a Lindelöf $G_{\delta}$-set. It follows from $\left[9\right.$, Theorem 10] that each $\widetilde{h}_{n}$ can be extended to a Baire-one function on $K^{\#}$. We will denote this extension by $h_{n}$.

Enumerate $H=\left\{a_{n} n \in \mathbb{N}\right\}$. As each $a_{n}$ is $G_{\delta}$, there is, for each $n \in \mathbb{N}$, a Baire-one function $u_{n}: K^{\#} \rightarrow[0,1]$ such that

$$
\left.u_{n}\right|_{K \# \backslash H}=1 \text { and }\left.u_{n}\right|_{\left\{a_{1}, \ldots, a_{n}\right\}}=0
$$

(see e.g. [9, Proposition 2]). Then $u_{n} h_{n}$ is a sequence of Baire-one functions pointwise converging to $\chi_{K}^{\#} \backslash H \cdot g$, which is therefore Baire-two.

As $g=\chi_{H} \cdot g+\chi_{K}^{\# \backslash H} \cdot g$ we conclude that $g$ is Baire-two. This finishes the proof for the case $\alpha=2$. 
The case $\alpha=1$ follows from the case $\alpha=2$. The case of general $\alpha \geq 2$ follows easily by transfinite induction.

Hence $g$ is of Baire class $\max \{\alpha, 2\}$ whenever $f$ is of Baire class $\alpha$. Now the function $x \mapsto \delta_{x}(g)$ is the required extension (where we again use [16, Theorem 3.3]).

Let us finish by asking the following natural questions.

Questions.

(1) Which of Theorems 1, 2, 3, 5, 6 have an analogue for general Choquet simplices?

(2) Suppose that each Baire-two function on $X$ can be modified to an affine Baire-two function coinciding with the original one on ext $X$. Can one choose such a modification to satisfy the barycentric formula?

(3) Suppose that each Baire-two function on ext $X$ can be extended to an affine Baire-two function. Can the extension be chosen to satisfy the barycentric formula?

Added in proof. J. Spurný and the author recently proved that Conjecture 1 is true in general.

\section{References}

[1] E. M. Alfsen, Compact Convex Sets and Boundary Integrals, Springer, 1971.

[2] H. Bauer, Šilowscher Rand und Dirichletsches Problem, Ann. Inst. Fourier (Grenoble) 11 (1961), 89-136.

[3] E. Bishop and K. de Leeuw, The representation of linear functionals by measures on extreme points, ibid. 9 (1959), 305-331.

[4] D. A. Edwards, Minimum-stable wedges of semicontinuous functions, Math. Scand. 19 (1966), 15-26.

[5] G. Fodor, On stationary sets and regressive functions, Acta Sci. Math. (Szeged) 27 (1966), 105-110.

[6] E. Hewitt and K. Stromberg, Real and Abstract Analysis, Springer, New York, 1969.

[7] P. Holický, Čech analytic and almost $K$-descriptive spaces, Czechoslovak Math. J. 43 (118) (1993), 451-466.

[8] F. Jellett, On affine extensions of continuous functions defined on the extreme boundary of a Choquet simplex, Quart. J. Math. Oxford Ser. (2) 36 (1985), 71-73.

[9] O. Kalenda and J. Spurný, Extending Baire-one functions on topological spaces, Topology Appl. 149 (2005), 195-216.

[10] K. Kuratowski, Topology, Vol. I, Academic Press, New York, 1966.

[11] J. Lukeš, J. Malý, I. Netuka, M. Smrčka and J. Spurný, On approximation of affine Baire-one functions, Israel J. Math. 134 (2003), 255-289.

[12] J. Lukeš, J. Malý and L. Zajíček, Fine Topology Methods in Real Analysis and Potential Theory, Lecture Notes in Math. 1189, Springer, 1986.

[13] J. C. Oxtoby, Measure and Category, 2nd ed., Springer, New York, 1980. 
[14] R. Pol, Remark on the restricted Baire property in compact spaces, Bull. Acad. Polon. Sci. Sér. Sci. Math. Astronom. Phys. 24 (1976), 599-603.

[15] M. Rogalski, Opérateurs de Lion, projecteurs boréliens et simplexes analytiques, J. Funct. Anal. 2 (1968), 458-488.

[16] J. Spurný, Representation of abstract affine functions, Real Anal. Exchange 28 (2002/2003), 337-354.

[17] —, Affine Baire-one functions on Choquet simplexes, Bull. Austral. Math. Soc., to appear; http://www.karlin.mff.cuni.cz/kma-preprints/.

[18] -, Affine Baire-one functions on compact convex sets, a preprint.

[19] P. J. Stacey, Choquet simplices with the prescribed extreme and Silov boundaries, Quart. J. Math. Oxford Ser. (2) 30 (1979), 469-482.

[20] F. Topsøe, Topology and Measure, Lecture Notes in Math. 133, Springer, 1970.

Ondřej F. K. Kalenda

Department of Mathematical Analysis

Faculty of Mathematics and Physics

Charles University

Sokolovská 83

18675 Praha 8, Czech Republic

E-mail: kalenda@karlin.mff.cuni.cz

Received January 27, 2005;

received in final form April 19, 2005 